\title{
Interaction Design and Usability of Learning Spaces in 3D Multi-user Virtual Worlds
}

\author{
Shailey Minocha and Ahmad John Reeves \\ Centre for Research in Computing, The Open University, Milton Keynes MK7 6AA, UK \\ \{S.Minocha, A.J.Reeves $\}$ @open.ac.uk
}

\begin{abstract}
Three-dimensional virtual worlds are multimedia, simulated environments, often managed over the Web, which users can 'inhabit' and interact via their own graphical, self-representations known as 'avatars'. 3D virtual worlds are being used in many applications: education/training, gaming, social networking, marketing and commerce. Second Life is the most widely used 3D virtual world in education. However, problems associated with usability, navigation and wayfinding in 3D virtual worlds may impact on student learning and engagement. Based on empirical investigations of learning spaces in Second Life, this paper presents design guidelines to improve the usability and ease of navigation in 3D spaces. Methods of data collection include semi-structured interviews with Second Life students, educators and designers. The findings have revealed that design principles from the fields of urban planning, HumanComputer Interaction, Web usability, geography and psychology can influence the design of spaces in 3D multi-user virtual environments.
\end{abstract}

Keywords: 3D virtual worlds, 3D virtual environments, design guidelines, Second Life, usability, wayfinding.

\section{Introduction}

Second Life (SL) is a persistent, online three-dimensional multi-user virtual world. Users synchronously interact in 3D spaces via their graphical self-representations known as 'avatars' and converse in real-time through gestures, and audio- and textbased (chat and instant messaging) communication [1]. Users connect to the SL environment with a software program called a client or viewer, which is responsible for displaying the 3D world and for negotiating user commands with a central server. Typically the client displays the user's avatar and surrounding portion of the world consisting of other avatars, landscape, buildings, etc. Unlike Massively Multi-player Online Role-playing Games such as World of Warcraft that have a scripted plot or storyline for the role-playing and game(s), SL, is not a 'game' per se. SL has a very strong user community, and the content and narrative is constructed and owned by the residents, rather than by Linden Labs, the company which provide the infrastructure, hardware and software to support SL.

In addition to social recreation and business applications, SL has attracted attention from academic institutions as an addition to face-to-face teaching or to be used in conjunction with 2D technologies such as blogs, wikis and discussion forums [2]. An 
island of an institution in SL can provide a dedicated environment for learning, which helps to ensure a sense of belonging and purpose for the students. The lack of a guiding narrative in SL provides flexibility for educators to design learning spaces for their pedagogical requirements. Although many educators have expressed the need for best practice design principles for creating learning spaces in SL, few have addressed the interaction design and usability of learning spaces in SL.

In the research project 'DELVE' (Design of Learning Spaces in 3D Multi-user Virtual Environments), we have conducted an empirical study involving SL educators, designers, and students to investigate their experiences with and perceptions of learning space designs in SL. In this paper, we focus on the empirical data related to the usability of learning spaces in SL. It is important to note that in our research that we have focused on the design of the 3D learning spaces within SL and we have not been concerned with the interface design and usability of the technology as such (i.e. SL client or viewer). Based on our empirical research, we present design guidelines or recommendations for educators and designers for designing usable learning spaces in $3 \mathrm{D}$ virtual worlds (VWs).

\section{Background}

In the disciplines of design, urban planning, and architecture, there are a number of studies that have investigated the design of 3D virtual environments (e.g. virtual reality and 3D VWs) in terms of navigation, orientation and wayfinding. In the late 1990s, the emphasis was to apply the understanding of navigation in the real world to designing usable interfaces in virtual environments (e.g. [3], [4]). Lynch [5] originally suggested that landmarks play a significant role in our cognition of the real world environment. Based on literature on navigation in real world including the work by Lynch, in [6] the author presents design guidelines for landmarks to support navigation in 3D virtual environments (VEs). A VE containing distinctive landmarks, edges and pathways supports navigation by facilitating the acquisition and application of spatial knowledge (e.g. see [7]).

Another important paper is that of Charitos [8] in which he proposes using Lynchlike components such as signs, landmarks, paths, places, intersections (nodes) and domains (districts) to aid navigation in VWs. However, the effectiveness of the wayfinding strategies on the users hasn't been tested and Charitos's approach is critiqued as being only conceptual [9]. However, in [10], the author (Dickey) has discussed that Charitos' pragmatic approach of aiding the navigation via environmental cues is most relevant to the design of educational environments. Dickey performed an empirical study involving the 3D world Active Worlds with her distance-education students to examine how architectural perspective of VW design, as described by Charitos, could be applied to provide design guidelines for creating 3D educational environments that reduce disorientation for users and aid wayfinding. However, Dickey [10] acknowledges the limitations of her approach in adopting the real world architectural perspective of designing 3D VEs: for example, real world concepts of weather and gravity have no impact in 3D VEs.

Although the mental models of real world navigation influence wayfinding and navigation strategies in 3D VWs such as SL, the ability to fly in SL and to teleport 
from one location to another within a space or island implies that not all of the architectural design guidelines from the real world can be applied to the design of SL spaces. Further, in SL, spaces may not always be replicas of real world places but may have varying degrees of visual realism, from photo-realism, or artistic realism, or metaphorical realism to having spaces that have elements of fantasy [11].

With regards to the design and usability of learning spaces in SL, the research has been mostly anecdotal and exploratory but increasingly students, designers and educators are discussing the significance of usability and the user experience on SL-related mailing lists and SL educators' events about the significance of usability and the user experience. For example, Barton Pursel (Penn State University, US) blogs on SL design and usability and makes some observations on usability issues such as navigation, space design and familiarity (see http://tinyurl.com/lm6dg7). Some consultancy companies (e.g. The Otherland Group) have looked at usability issues in virtual worlds (see http://tinyurl.com/59ce9d). They carried out an exploratory study that focused on customer experience and issues such as trading, branding and customer retention. However, their study highlights the need to follow generic Human Computer Interaction (HCI) principles and adopt a user-centred design approach to the design and evaluation of SL spaces: e.g., designing for the avatars (users) and not humans; and providing orientation to the user ("where am I?"; "what can I do here?"; "where can I go from here?"; "where have I been?”; and "how do I get back?”).

John Wallace, an SL educator, discusses usability of learning spaces in SL by applying web usability principles. His blog and wiki are at: http:// instructionalalchemy.com/blog/ and http://tinyurl.com/lg65od, respectively. Other SL users have begun to look at usability in terms of accessibility (for example, the SL group 'Virtual Abilities') by blending universal design principles with webaccessibility guidelines; initiatives include accessibility of SL for the visually impaired and mobility impaired. There are, however, no empirical studies to-date that we are aware of which relate to the design and usability of learning spaces in SL or design of SL spaces, in general. Therefore, when institutions aspire to create learning spaces in SL, there are few studies or guidelines to inform them except for individual case studies (e.g. [12]). In this paper, we report on a study in which we have elicited educators', designers' and students' perceptions of the usability of learning spaces within SL. Based on this empirical research, we have derived design guidelines for educators and designers who are involved in designing learning spaces in SL. Although we have focused on SL learning spaces, we hope that the results will be applicable to design of SL spaces, in general, and to other avatar-based 3D VWs.

\section{Research Design}

The key research question in our research project DELVE has been "how should 3D learning spaces be designed for student engagement?" We have been primarily concerned with the realism of learning spaces in SL: whether and how does the realism or non-realism of learning spaces influence student learning and engagement. However, in our empirical investigations, we found that interaction design and usability of 3D learning spaces were considered to be key factors that influence student experience. In 
this paper, we report the analysis of a subset of DELVE's data that is related to the users' perceptions of usability of learning spaces in SL.

\subsection{Data and Methodology}

Our research methodology consisted of an online survey conducted at the start of the project involving colleagues from UK's further education (FE) and higher education (HE) communities. This was followed by semi-structured interviews with designers, educators and students. The various stages of our empirical research were as follows:

We began by developing and conducting an online survey using the Survey Monkey application. The aim of the survey was two-fold: firstly, to ask a range of questions relating to the design of learning spaces in SL from colleagues in the FE and HE community. Secondly, we wanted to invite the survey participants to take part in a follow-up interview. We received 46 filled questionnaires and 27 respondents expressed willingness to participate in an interview. In parallel to conducting the survey, we developed our research materials such as a consent form, project summary sheet, pre-interview questionnaire and interview templates. The educators' and designers' questions related to the description of learning spaces, factors affecting designs, levels of realism, and learning activities. The students' questions covered similar issues along with an emphasis on the design aspects that either supported or hindered their learning experiences. Our institution's Ethics Committee reviewed and approved the research materials prior to our carrying out the interviews.

From the 27 survey participants who had agreed to participate in interviews, we selected 15 participants and sent out email invitations. Other modes of participant recruitment were: notices in some of the education groups within SL and personal invitations to colleagues. In order to evaluate and improve our research materials, we carried out pilot interviews. In all, we interviewed 7 students, 10 designers and 22 educators. Four interviews were conducted over the phone (audio recorded) while 35 interviews were conducted in SL and transcripts were saved into individual files.

Our research question provided the lens to analyse the data from the participants. An inductive or thematic analysis of the data was undertaken by us (the project team) to identify the themes, sub-themes, and any causal or inter-relationships between the themes [13]. The inductive analysis involved two team members reading the interview accounts. After an independent data analysis, the team focused on finding recurring themes in the analysed data.

\section{Main Findings of the Study}

We now report the data analysis from the core subset of the data related to interaction design and usability. For each of the derived themes, vignettes (quotes) from the interviews are included. In fact, each of the themes and sub-themes are guidelines for designing learning spaces in SL.

Design for affordance of learning spaces and objects or as per real world conventions within learning spaces: Affordance is a property in which the physical characteristics of an object or environment influence its function. When the affordance of an object or environment corresponds with its intended function, the design will perform 
more efficiently and will be easier to use. In Figure 1(a), the postbox or a drop box, a familiar object from real world, is placed for receiving feedback from students and other visitors on the island.

One of the participants discussed that the design of the learning space should indicate the type of learning activity that can be carried out in that space: "depending upon what it is you want to do, the space may need to look different in order to function as you need. For example, row of desks with a lecturer in front is perfect for demonstrations and giving important information, whereas the seminar table is great for discussions and sharing ideas with each other. The informal areas on the sim [island] also show that any space can be a meeting area, just a nice place to meet and talk and reflect" [SL designer].

An educator discussed how the intended function should be integrated within the design: "I say one thing it needs to be very clear what the aim of the space is, ... that should not be because there is a nice sign saying what it is for but it should be in the character of the development. Something in the look and feel, the way you interact with the space is representative of its use so that it will make it easy for the user to understand" [Deep Think]. The objects in the learning spaces should imply the way in which the spaces can be used: "Well, I already mentioned chairs, although totally unnecessary [for the avatars] they do signal that a meeting is going on and certain people have committed to participating for a while" [SL educator and designer]. In Figure 1(b), chairs, a table, slide presenter, posters on the walls have been used to aid a presentation and follow-on discussions.
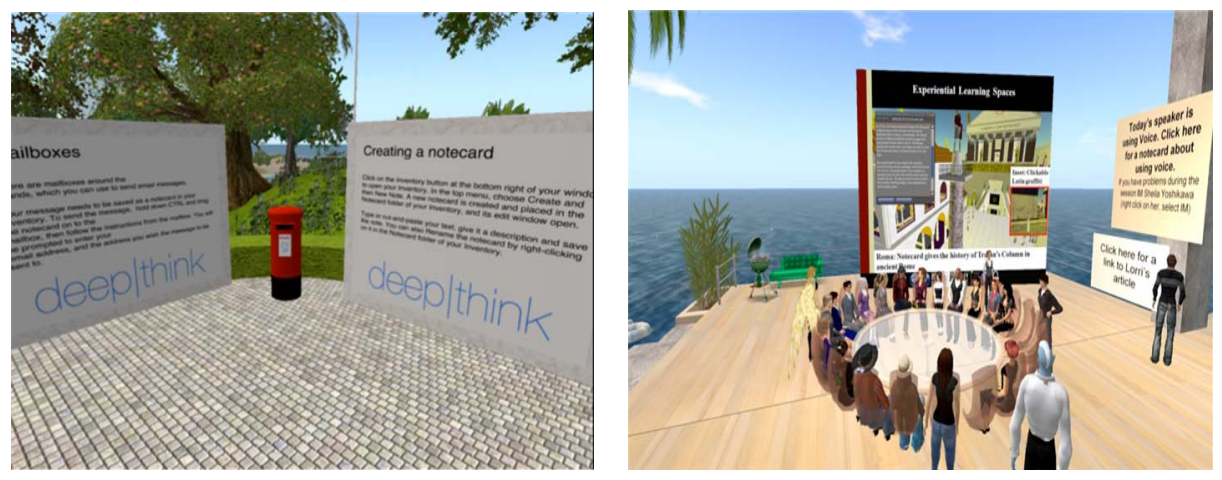

Fig. 1. (a) A drop box to collect feedback; (b) A round-table discussion

Design for storytelling: Storytelling involving presenting information in a way that will help engage an audience in a design or provide a rich context to enhance learning In our study, both educators and designers mentioned about having a story or narrative for the space (as if staging a play): "the space is like a story, you need to involve the space". Linking the structure of the space and narrative enhances learning.

This educator also suggested that how scenarios or stories of usage of the space could also be an effective means of communicating within a multi-disciplinary design team. The educator developed user scenarios to understand how the spaces would be used; the scenarios were used in discussions with developers and also to create tutorials: "At some points they [developers] couldn't understand what the problem was or 
what we were trying to achieve so we came up with user scenarios...we adapted scenarios used in software engineering for a user journey through the island... and I finally understood the way we were thinking the way students would go round this campus and use the space..."

Use real world metaphors: Metaphors may be considered as tools or processes that enable understanding of one thing in terms of another [15]. In our study, educators and designers mentioned about using familiar metaphors from the real world, for example, use of mailboxes in SL spaces where students could leave messages, or having search pods in the library, familiar seating (like in the real world), and buildings (see Figure 2(a)).

Consider ambience and aesthetics of the learning space: Ambience and visual aesthetics are important design criteria. Aesthetic designs are perceived as easier to use and promote creative thinking and problem solving (see Figure 2(b)): "If they feel relaxed here, from visually stimulation, or aural (sound of water flowing)...then maybe they engage more with the tutorial and say more than they would in real-life in lecture hall or for something comparable with MSc usage - they are distance learners and we use discussion boards, skype, wikis, blogs, email...” [SL educator].
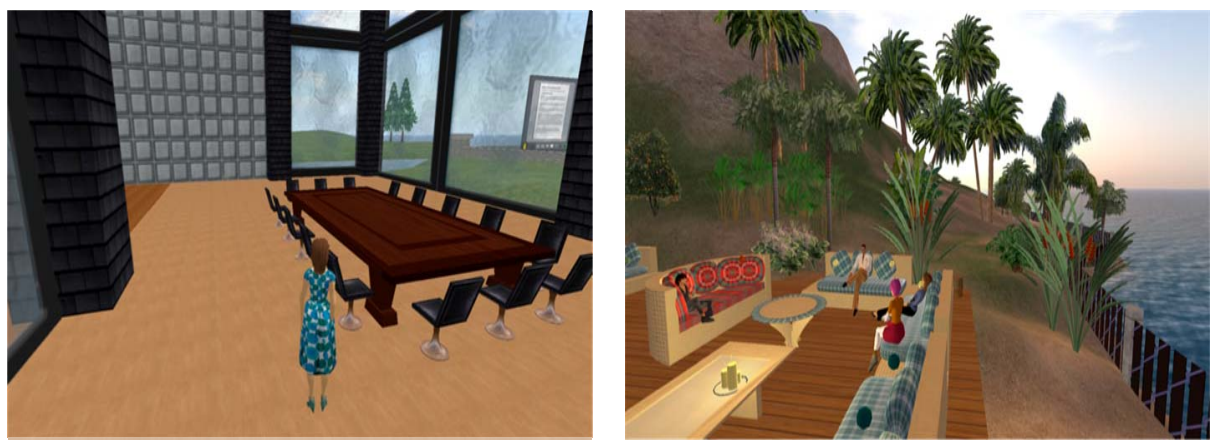

Fig. 2. (a) A meeting room; (b) An open-air meeting venue

Consider realism for familiarity and comfort: A related design principle to affordance is designing visually realistic learning spaces, especially for users who may be new to SL. Visual realism, that is depicting objects and spaces as they are in real world (e.g. lecture rooms with tables, chairs and a podium) helps to support existing mental models of the learners of what to expect [11], and how to interact or behave (e.g. Figure 2(a)): "I think having visual realism helps people feel comfortable with the environment, and if they are comfortable then you can extend the boundaries of the activities a bit further..." [SL designer]. An educator said "...some level of visual realism provides clues to a person on how they might behave."

Design to orient the user at the landing or entry point: The initial impression of a system or environment greatly influences subsequent perceptions and attitudes, which then affects the quality of subsequent interactions. The entry points or points of prospect are points of physical or attentional entry into a design and should allow users to become oriented and clearly survey available options. 
When a student decides to visit a SL learning space, they will normally teleport to the space's arrival point via a 'SLURL' (a direct teleport link to a location in SL), either from the web browser, or from another SL location. Upon arrival in Deep Think, one of the islands of SL, the user is presented with a 3D map of the island showing what is there and how they are located in relation to one another (see Figure 3(a)).

In addition, entry points should have progressive lures that attract and pull users through the entry point. In Deep Think, there are information boards and a video that discusses the purpose of the island and its various learning spaces. There are boards which give details of individual learning spaces space (study area, auditorium, etc). There is a teleport board (map of the island) with provision to click on a specific place on the map and to move to that area (see Figure 3(a)). Some learning spaces or islands provide notecards on arrival with details of the island and how the spaces can be used.

Taking the example of webpage 'stickiness' i.e. the attribute of a site to keep users there and clicking more web pages, SL 'location stickiness' can be enhanced through a well-designed and appropriate arrival point. As one designer commented: "An island has elements of real spaces but also has elements of web-pages. People can be channeled to arrive at a specific point (like with an home page) and this orientation space can be used to provide initial information, engage the user and to show them paths further into the function/space. People are impatient just as they are with web pages. Make them walk everywhere and they won't bother looking. Give them a way to click something and be immediately transported to another part of the space, and they are much more likely to explore deeper." [SL designer]. In Figure 3(b), a tour facility at the arrival point takes the user through the various places on the island to orient and inform the user through a textual commentary.
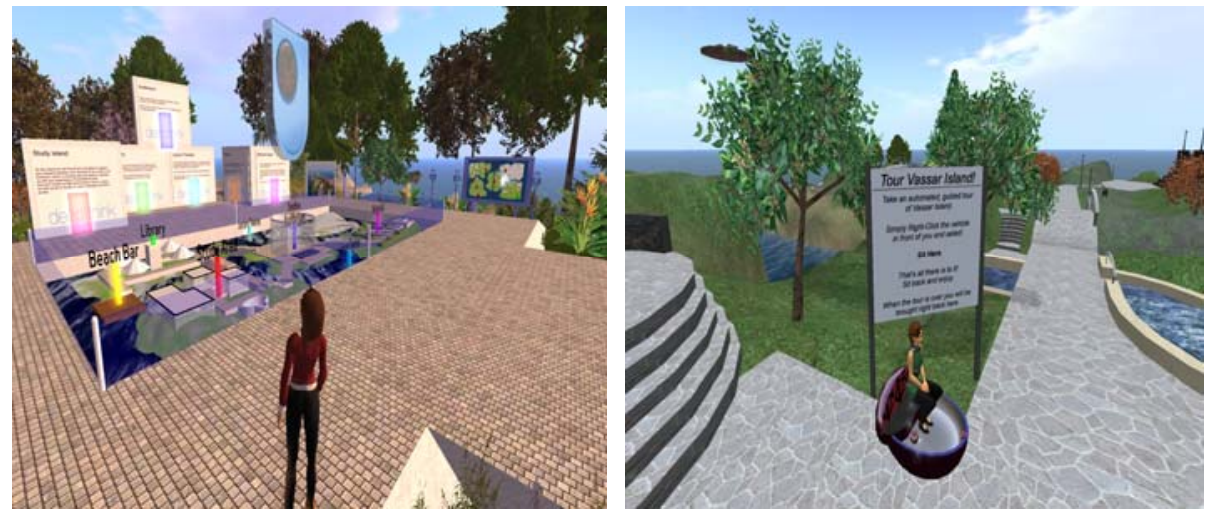

Fig. 3. (a) An entry point in Deep Think; (b) Tour in Vassar island

A related aspect to entry point is 'wayfinding'. When examining how an architectural perspective of virtual world design may provide guidelines for creating educational virtual environments, Dickey [10] highlighted the advantages of landmarks, signs, paths and boundaries in 3D spaces to support wayfinding. Providing teleport maps or even maps (see Figure 4) help in orientation and provide robust mental representations of the space and aid the user in route decision-making. 

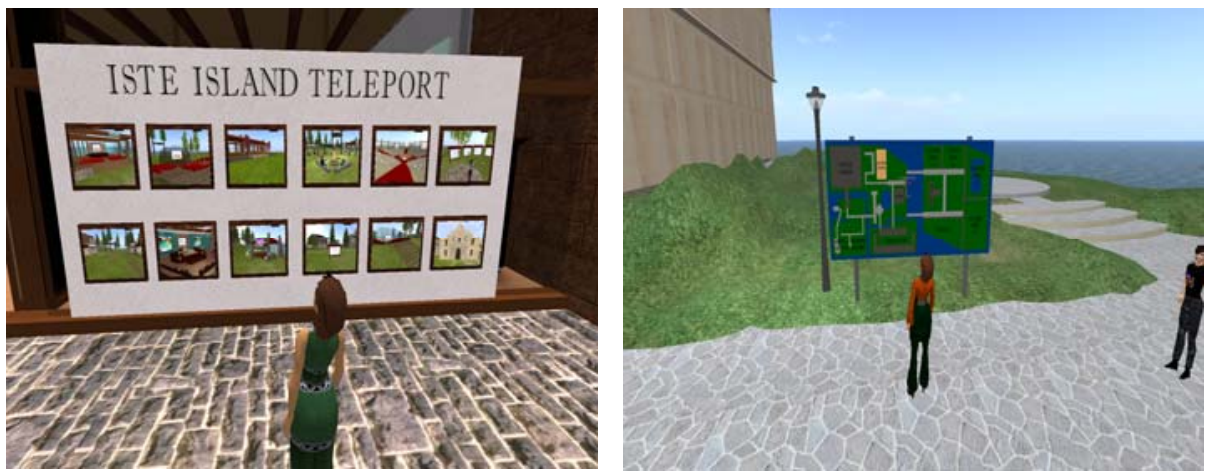

Fig. 4. (a) A teleport map; (b) map showing the various places on the island

Signs and paths help the user to navigate to their destination: “...students need certain reference points when they are entering an unfamiliar space... for example, we created definite pathways through the exhibition and some signposts and a welcome message but after that it is up to the student to explore - a few sign posts to help them ground themselves. Especially for newbies it can take a while to learn to control your avatar and I have been to spaces where I got trapped in labyrinth type buildings the only way out was to teleport" [SL educator]. Figure 5(a) shows signage which is similar to real world signage. In Figure 5(b), each path has an arch with the destination's name at the top of the arch, thereby providing a clear route. Along with navigation to areas or buildings comes the issue of navigating within areas or buildings. Avoiding confined spaces and unnecessary objects all help the user to move freely.
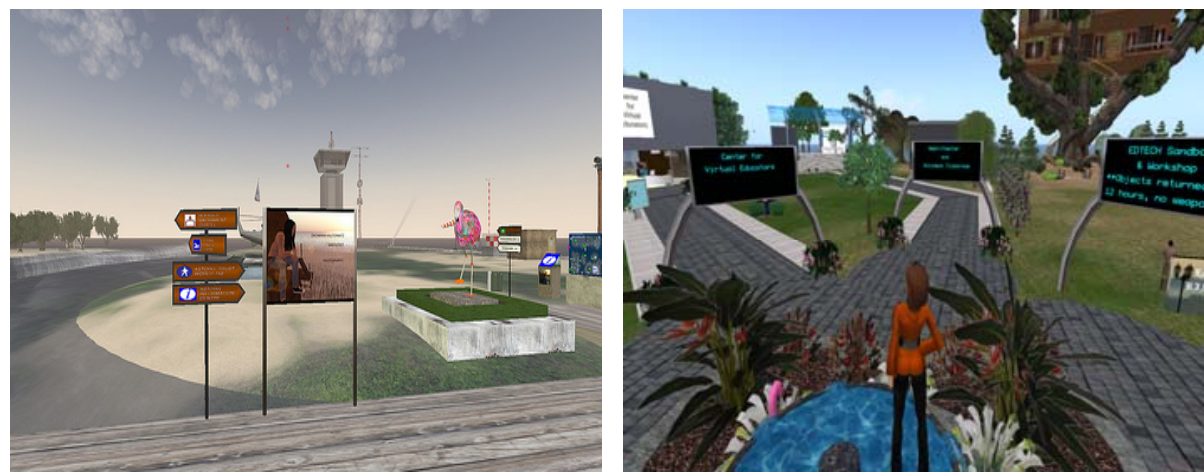

Fig. 5. (a) Signage to guide navigation; (b) Paths to aid route decision-making

Avoid spaces that can trigger phobic reaction or don't provide an easy exit. An educator and SL designer discussed her experiences of interacting in confined spaces in SL (note that these interactions are via avatars): "The spaces in the brownstone [a learning space replicating the era in 1920s-30s] are pretty much consistent with reallife... I find that a problem there as well as some of the other builds I've been in. 
I suffer from vertigo and the store is on four floors in relatively confined spaces and as I was travelling up and down furnishing the areas I found it was triggering my vertigo. So I put in teleporting equipment not yet available in real-life or the 1920's or 30's!' [SL educator and designer]. Figure 6(a) shows a space which has no open window or an exit (the only way to exit is to teleport). The avatars lose their camera/views behind the walls or outside the meeting room. This space made the educators (whom we had taken on a tour in our study) very claustrophobic and uncomfortable.

An SL designer said: "I try to steer away from having buildings with roofs as they are actually unnecessary in SL and they mess with people's camera angles". Thus, buildings in SL should be as open as possible: avoiding closed roofs, annoying doors, narrow corridors or cluttered areas that can both constrict avatar movement and cause lag. Clear options and signage to exit buildings should be provided such as through non-solid walls, open roofs and spaces, clear exit signs etc.

Form should follow function: This principle implies that aesthetic considerations in design should be secondary to functional considerations. The participants mentioned that the design should reflect the learning activities: "... the design should be functionally and pedagogically appropriate, [and] integrated'... a design for a purpose”. One educator gave a specific example: “... I actually used objects that looked like books, so the student would go up to a book and the book would open for them ..., so I tried to keep that form and function consistent with real-life and that sort of worked quite well" [SL educator] (see Figure 6(b)).
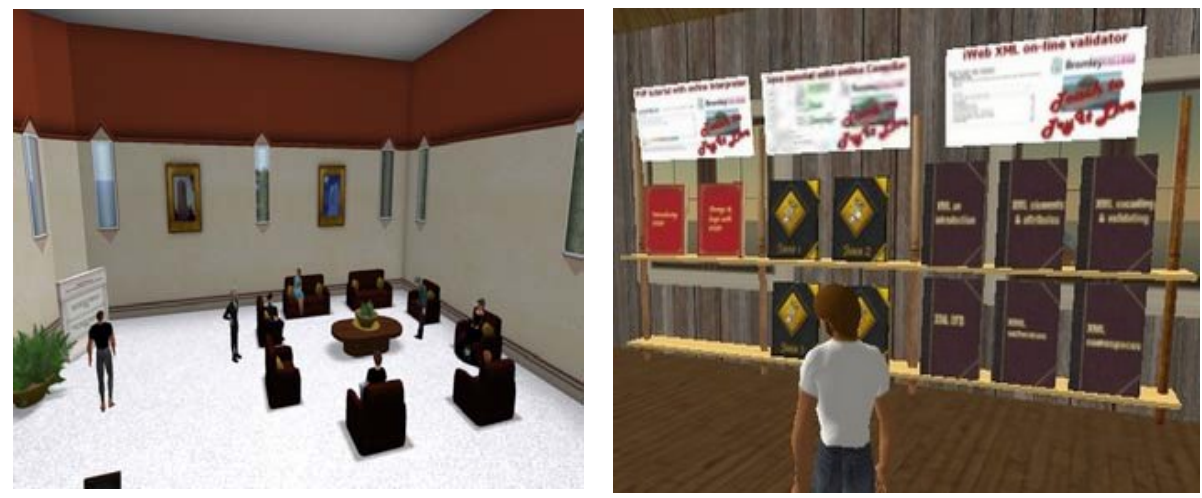

Fig. 6. (a) Room without a normal exit; (b) Metaphors for conveying the function

\section{Discussion and Conclusions}

The subset of data and findings presented in this paper has revealed that applying architectural principles of real world designs to 3D virtual worlds as discussed by Charitos [8] and Dickey [10] may not be sufficient. In fact, we have seen that design principles from the fields of urban planning, HCI, Web usability, geography and psychology influence the design of spaces in SL. The design guidelines and vignettes presented in this paper will provide useful guidance and triggers for ideas to educators and designers who are planning to set up learning activities and spaces in SL. 
In our study, we have noted that educators and designers are clearly taking advantage of the 3D features of SL and its interactivity and flexibility for designing (and redesigning). They are adopting a user-centred design (UCD) approach and the designs of learning spaces are changing and evolving through an iterative UCD and evaluation process.

Our research in the DELVE project has shown that designs of learning spaces in SL may influence student learning and engagement. However, there are several other contextual factors that may impact on student experience: such as student's SL skills, their motivation, and the educator's SL skills and preparations for the activities, whether SL is a compulsory component, whether SL activities will be assessed; and the nature of course delivery (distance education, face-to-face, or blended delivery).

Limitations of our empirical investigations: We interviewed a small number of students as compared to educators and designers in our empirical research. Furthermore, there is a need for conducting longitudinal studies where we can capture users' experiences over a period of time. This would enhance our understanding of the interrelationships between learning experiences and the designs of the learning spaces as the designs evolve over time. Finally, we haven't (to-date) evaluated the design guidelines proposed in this paper for their applicability and usefulness.

Taking this research further: There are three key areas that we hope, will contribute towards a better understanding of the design of learning spaces in 3D VWs: (a) it would be useful to draw out lessons from the design of physical learning spaces [16], and particularly, principles of accessible physical designs (b) The principles of game usability (e.g. [17]) will help enrich the designer's toolbox for designing and evaluating 3D learning spaces for immersion, fun, flow, playfulness, choreography, and engagement; (c) principles of cross-cultural usability. The signs for wayfinding and navigation, the metaphors in the design of objects and the islands, and the symbols and icons used are some of the design aspects, which can be country and culturespecific. As with websites and designs of other products and services, it will be useful to develop guidelines for cross-cultural usability for designing SL spaces.

\section{Acknowledgements}

The research presented in this paper has been supported by JISC, Faculty of Mathematics, Computing and Technology of the Open University, UK, and Teaching fellowship from Centre for Open Learning of Mathematics, Science, Computing and Technology, one of the Centres for Excellence in Teaching and Learning at the Open University, UK. We would like to express our sincere thanks to all the participants in our study.

\section{References}

1. Meadows, M.S.: I, Avatar: The culture and consequences of having a Second Life. New Riders, California (2008)

2. Minocha, S., Roberts, D.: Laying the groundwork for Socialisation and Knowledge Construction within 3D Virtual Worlds. ALT J. 16(3), 181-196 (2008) 
3. Ruddle, R.P., Payne, S.J., Jones, D.M.: Navigating buildings in "desk-top" virtual environments: Experimental investigations using extended navigational experience. J. Experimental Psychology 3(2), 143-159 (1997)

4. Darken, R.P., Banker, W.P.: Navigating in natural environments: A virtual environment training transfer study. In: IEEE Virtual Reality Annual International Symposium, pp. 12 19 (1998)

5. Lynch, K.: The Image of the City. MIT Press, Cambridge (1960)

6. Vinson, N.G.: Design Guidelines for Landmarks to Support Navigation in Virtual Environments. In: Proc. CHI 1999, pp. 278-285. ACM Press, New York (1999)

7. Steck, S.D., Mallot, H.A.: The Role of Global and Local Landmarks in Virtual Environment Navigation. Presence 9(1), 69-83 (2000)

8. Charitos, D.: Designing Space in virtual environments for aiding wayfinding behaviour. In: Proceedings of the 4th UK Virtual Reality SIG Conference, Brunel University (1997)

9. Conroy-Dalton, R.: Is spatial intelligibility critical to the design of large scale virtual environments? International Journal of Design Computing 4, 1329-7147 (2002)

10. Dickey, M.D.: An architectural perspective for the design of educational virtual environments. J. Visual Literacy 24(1), 49-66 (2004)

11. Minocha, S., Reeves, A.J.R.: Design of Learning Spaces in 3D Virtual Worlds: An Empirical Investigation of Second Life. J. Learning Media and Technology, accepted and to appear in the special issue on Learning in Virtual Worlds for Learning, Media \& Technology (2010)

12. Lucia, A.D., Francesse, R., Passero, I., Tortora, G.: Development and evaluation of a virtual campus on Second Life. Computers \& Education 52(1), 220-233 (2009)

13. Braun, V., Clarke, V.: Using thematic analysis in psychology. Qualitative Research in Psychology 3, 77-101 (2006)

14. Lidwell, W., Holden, K., Butler, J.: Universal Principles of Design. Rockport, Inc. (2003)

15. Lakoff, G., Johnson, M.: Metaphors we live by. The University of Chicago Press (2003)

16. Oblinger, D.: Learning Spaces. EDUCAUSE, London (2006)

17. Isbister, K., Schaffer, N.: Game Usability: Advice from the experts for advancing the player experience. Morgan Kaufmann Publishers, San Francisco (2008) 